\title{
Gradual acquisition of visual discrimination tasks in a social group of rhesus monkeys (Macaca mulatta)
}

\author{
CHRISTINE M. DREA and KIM WALLEN \\ Yerkes Regional Primate Research Center, Lawrenceville, Georgia \\ and Emory University, Atlanta, Georgia
}

\begin{abstract}
An established, captive colony of 74 rhesus monkeys (Macaca mulatta) was group-tested on a simultaneous visual discrimination problem and three reversals of the initial discrimination. The task incorporated important aspects of rhesus foraging behavior. Although other studies of communal groups of nonhuman primates have reported rapid learning, subjects in the present study showed no evidence of one-trial acquisition of the initial problem or of rapid learning-set formation across the reversal series. Instead, mean and individual performance, on all variables measured, improved gradually, both within and across series. Subjects appeared to "learn how to learn," consistent with findings of traditional studies of individual discrimination learning. Our finding of gradual learning in group-living animals argues that the source of rapid learning in previous reports is not attributable to social influence or ecological relevance, but may reflect specific procedural or species differences between studies.
\end{abstract}

The comparative study of learning has used two broad approaches: a laboratory approach concerned with the mechanisms of learning, and an ecological approach concerned with functional significance. Studies of nonhuman primate discrimination learning have relied primarily on the former, investigating the behavior of individuals under the controlled and isolating conditions of a standardized apparatus. This traditional approach consistently has shown that performance gradually improves, within and across discrimination problems, as individuals either acquire problem-solving strategies or learn to successfully apply strategies to new problems (Harlow, 1944, 1949). This "learning how to learn," termed learning-set formation, is considered a fundamental characteristic of discrimination learning.

The validity of this traditional approach has been questioned because it does not adequately duplicate ecological demands (Johnston, 1981, 1982; Kamil \& Yoerg, 1982;

This article is based on a portion of a thesis submitted to Emory University in partial fulfillment of the requirements for the master's degree. Support was provided by National Institutes of Health Grant RR-00165 from the Division of Research and Resources to the Yerkes Regional Primate Research Center and by National Science Foundation Grant BNS86-07295 to K. W. The Yerkes Regional Primate Research Center is fully accredited by the American Association for the Accreditation of Laboratory Animal Care. We thank Irit Gat, Lisa Friedberg, and Deanna Gill for their assistance with data collection, and John Sikes for constructing the apparatus. Harold Gouzoules, Michael Tomasello, Michael Zeiler, and Thomas Gordon are gratefully acknowledged for their helpful suggestions. Correspondence concerning this article should be addressed to C. M. Drea, Department of Psychology, University of California, Berkeley, CA 94720-1650.

-Accepted by previous editor, Vincent M. LoLordo
Shettleworth, 1984). Notably, individuals are divorced from their social context, and tasks bear a weak relation to problems routinely encountered in the natural habitat. Thus, although the performance of individuals under artificial testing conditions demonstrates the potential of nonhuman primates to use gradual learning and develop learning sets, this may not be relevant to subjects under more natural conditions (Gould \& Marler, 1984; Jay, 1965; Johnston, 1981; Seligman, 1970). These issues have led to an increased effort toward incorporating ecological concerns into laboratory paradigms (Timberlake, 1990).

Only a few discrimination studies have adopted an integrated approach by testing individuals while in their social group and by reinforcing responses that occur in the natural context of the animal's behavior. Notably, these studies produced results that challenge the notion of learning-set formation. For instance, Menzel and Juno (1982) reported one-trial visual discrimination learning of a successive object discrimination task by a family group of marmosets (Saguinus fuscicollis). They found that a single exposure to objects associated with food increased the frequency of approach to those objects on the next trial. Thus, neither repeated exposure to stimulus objects nor formal training was necessary for subjects to show evidence of acquisition.

On the basis of their data, Menzel and Juno (1982, 1984) questioned the significance of learning-set formation and proposed that the slow rate of learning by individuals reflects the irrelevance of the traditional testing environment. Others, however, attributed their finding of rapid learning to procedural differences (Schrier, 1984; Schrier \& Thompson, 1984). Specifically, the use of a successive discrimination paradigm arguably fails to test for learning-set formation because it does not allow 
the application of a "win-stay, lose-shift" strategy (Levine, 1959). Furthermore, use of "group proximity" (i.e., the number of subjects approaching objects in 15sec intervals) as the dependent measure of discrimination acquisition, rather than the traditional variable of individual response and reward retrieval, failed to provide information on individual patterns of learning.

Lepoivre and Pallaud, studying discrimination learning in a small group of Guinea baboons (Papio papio), found that different dependent variables showed different patterns of learning (Lepoivre \& Pallaud, 1985, 1986; Pallaud \& Lepoivre, 1985). Using a simultaneous presentation paradigm, group-tested subjects had to differentiate baited from nonbaited caches on the basis of an associated color cue. Preferential searching in a baited area, regardless of reward retrieval, showed gradual improvement across trials, consistent with learningset formation. In contrast, group proximity to the baited cache showed one-trial associative learning, in agreement with Menzel and Juno (1982). However, this evidence of one-trial learning occurred during a spatial discrimination task that followed a series of habituation, color discrimination acquisition, and discrimination reversal trials. Thus, this result could also be evidence of rapid learning-set formation culminating in one-trial learning. Nevertheless, group proximity showed more rapid learning than did individual behavior, demonstrating the importance of choice of dependent variable in learning studies.

In short, though these two discrimination studies using social primate groups suggest different patterns of learning from traditional studies of single subjects, they produced inconsistent results. The purpose of the present study was to further examine the effects of social groups on discrimination learning in primates. We tested members of a large captive troop of rhesus monkeys (Macaca mulatta) on a series of color discriminations, using a simultaneous presentation paradigm and a task that incorporated important aspects of foraging behavior.

Primates rely on colored nonfood items as signs of potential food availability (Jarvik, 1953; Menzel, 1974), confirming that color is a prepotent cue (D'Amato \& Fazzaro, 1966; De Valois \& Jacobs, 1968; Devine, 1970; Snodderly, 1979). Although the ability to discriminate between qualitatively different food signs is relevant to environmental demands, the natural conditions under which such associations occur are frequently inadequately replicated in the laboratory. For instance, accurate discrimination by a forager does not always result in obtaining food, whereas correct test responses are usually rewarded. Similarly, the depletion of natural food sources is rarely duplicated experimentally, because rewards are constantly replenished. Also, the uncovering of hidden food, an integral feature of foraging (Jolly, 1985), requires both visual and manual discriminations, whereas usually only one mode is studied in the laboratory.

In the present task, not all responses following accurate visual discrimination were rewarded. This is because the probability of obtaining a reward decreased as a function of the number of rewards previously obtained by others and because successful performance involved both visual and manual discrimination. The task was also presented in an ecologically relevant social context, as group size, sex ratio, and age distribution were consistent with those of free-ranging troops (Lindburg, 1971). Furthermore, group testing in the home compound allowed social affiliations to remain intact. We investigated the pattern of learning during an acquisition and a reversal phase, and we assessed the effect of social context on a variety of measures previously used to demonstrate gradual or one-trial learning.

\section{METHOD}

\section{Subjects and Housing}

The subjects were a captive colony of 74 rhesus monkeys, including 49 females and 25 males, ranging in age from 1 to 17 years. All were born and reared socially at the field station of the Yerkes Regional Primate Research Center of Emory University, in Lawrenceville, Georgia. The colony included members of 6 matrilines and 9 non-natal adult males. Matriarchs and their progeny had lived together for over 15 years and maintained a stable social structure. The subjects were housed and tested in a $38 \times 38 \mathrm{~m}$ outdoor compound. Outside of testing, the subjects had access to a temperature-controlled indoor area. The animals were provided with monkey chow before and after testing, oranges were provided after testing, and water was freely available. All subjects were dye-marked, for individual recognition during videotaped behavioral observations, using an analine dye (No. 1 Nyanzol D. Flakes No. 21707).

\section{Discrimination Task, Apparatus, and Testing Arena}

The subjects were presented a simultaneous visual discrimination problem, followed by three reversals of the initial discrimination. The apparatus was a set of green and a set of orange closed metal boxes, installed on the fenced enclosure in two experimental zones (Figure 1). To investigate box contents, the subjects had to climb the fence and insert an arm through the wire mesh into the central box opening.

Each color, in turn, signaled the presence or absence of limited rewards (i.e., peanuts). "Stocked" boxes of the designated correct color were provisioned with peanuts hidden in a sand-and-stone matrix, so that reward retrieval required manual discrimination between peanuts and similarly sized stones. Rewards were not replenished after removal during a single trial. "Nonstocked" boxes of the incorrect color contained only sand and stones. Stones were in all boxes to prevent the presence of an object from serving as a cue for peanut availability. Color location changed between zones according to a randomized sequence, resulting in locating the reinforced color in the left zone on 46 trials and in the right zone on 45 trials.

\section{Procedure}

Habituation. During a habituation series, with one $30-\mathrm{min}$ trial per day, the subjects were trained to run indoors on cue and were accustomed to the apparatus and testing conditions. While the subjects were sequestered indoors, each box was provisioned with 20 peanuts and 5 stones, for a total of 160 available peanuts per trial. Additionally, a small quantity of sand ( 0.5 liter) was added, cumulatively across trials, until a final depth of $10 \mathrm{~cm}$ was obtained. Thus, peanuts were initially visible but were eventually completely buried, and substantial effort was required for their retrieval. Once the boxes were installed, the subjects were released from, and locked out of, the indoor facilities, signaling the start of a trial. After testing, the subjects were again sequestered while the boxes were removed and their contents tallied. Habituation ended after 


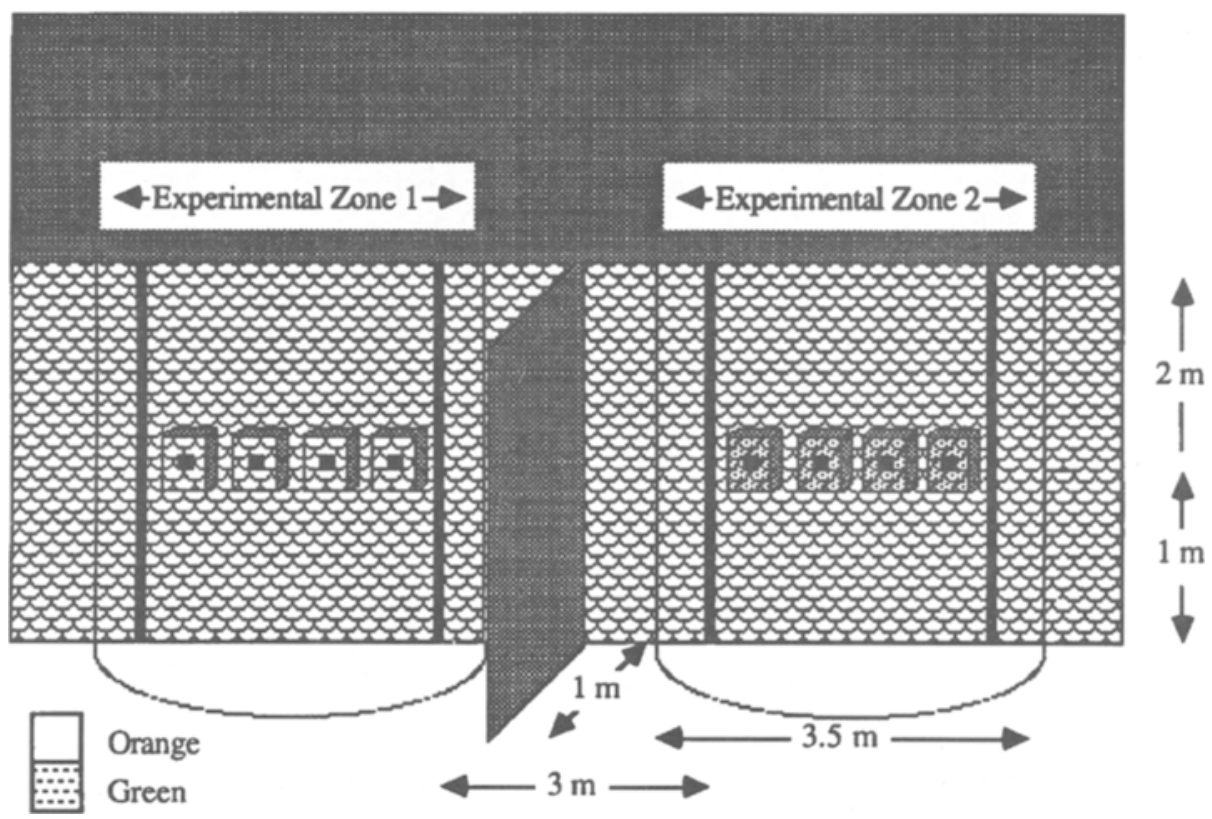

Figure 1. Apparatus and testing arena (not drawn to scale). Each box (31 $\mathrm{cm}$ wide $\times 37 \mathrm{~cm}$ high $\times 21$ cm deep) had a removable lid for provisioning, a metal hook on either side for attachment, and a central opening $(8 \times 8 \mathrm{~cm})$ for manual investigation. Boxes, grouped by color, were installed on the outside of the fence, with their openings facing the compound. The fencing surrounding each set and the ground below each set constituted two experimental zones (delineated by thin lines). Within sets, boxes were separated by $35 \mathrm{~cm}$. Sets were separated by at least $3 \mathrm{~m}$ and by an opaque metal barrier that stood at a right angle to the fence to block visual access between zones.

16 trials, once the subjects readily searched all boxes and retrieved over $90 \%$ of the rewards.

Initial discrimination series. During an acquisition series, green boxes were designated as "correct" and were the only ones stocked. There were two or three 20-min trials per test day, with a minimum of $1 \mathrm{~h}$ between trials. Fifteen peanuts and 10 stones were buried in each stocked box, for a total of 60 available rewards per trial, and 25 stones were buried in each nonstocked box. Usually, discrimination acquisition is determined by an a priori performance criterion; however, because of the complexity of the testing situation, performance could only be evaluated after lengthy coding completed after testing. Therefore, we used the retrieval of at least $90 \%$ of the rewards, over four consecutive trials, as a simple "group" measure of acquisition. This criterion was met in 25 trials.

Diserimination reversals. The initial discrimination series was followed by a discrimination reversal problem, for which the contingencies changed so that the orange cue signaled rewards and the green cue signaled no rewards. This first reversal was followed by two more reversals, producing two problems for each set of contingencies. Procedures were the same as for the initial discrimination. Following the same "group" acquisition criterion, the three reversals ran for 24,23 , and 19 trials, respectively.

\section{Data Collection}

The study ran through the nonbreeding season and ended after the third reversal to avoid introducing confounds resulting from the dramatic rise in sexual behavior that occurs with the onset of the breeding season. We collected detailed data for the discrimination and reversal series, but not the habituation series. Observations occurred between 0900 and 1200 h from a nearby tower. One observer, paying attention to reward retrievals that were visible with binoculars, reported behavior to a second observer, who then re- corded data on a portable computer that time-tags each entry with an accuracy of $1 \mathrm{sec}$. In addition to real-time data collection, each zone was videotaped by a separate camera. After completion of the study, tapes were viewed, frame by frame, by the first observer to retrieve any missed behavior. Data were transferred from the portable computer to a mainframe computer for editing, validation, and extraction. One trial was scored twice to determine intraobserver reliability, with agreements exceeding $95 \%$ for all measures. Another trial was scored by a third observer to determine interobserver reliability, with agreements exceeding $80 \%$.

Performance measures included the number of subjects entering zones, their behavior while in zones, and their ultimate success. The first measure (i.e., group proximity) was comparable to Menzel and Juno's (1982) measure and, though monitored in real time, was calculated at 15-sec intervals for purpose of comparison. Individual behavior (i.e., response allocations between zones) was scored both as duration measures, including time in zones and on boxes, and as frequency measures, including zone entries, box visits, and manual searches. Manual search was scored in bouts, defined as one or more consecutive reaches into a box, terminated by pauses of at least $30 \mathrm{sec}$ or by leaving the box. Manual search, although most directly comparable to Lepoivre and Pallaud's (1986) behavioral measure, underrepresented group preference since more animals were likely to enter zones rather than search boxes. Therefore, the complete behavioral repertoire was included to test for differences in susceptibility to social influences. Finally, the last measure was the number of rewards retrieved and was comparable to the traditional measure of success.

\section{Analyses}

Because of the lengthy coding process, selected trials were transcribed and data are presented for those trials only. For the initial discrimination series, the first 5 (early) and the last 5 (late) trials 
were scored to provide information on acquisition. For each reversal, 12 trials were scored. To test for progressive error reduction within and across reversals, the focus of analysis was on early trials (i.e., Trials 1-6), with a sampling of later trials (i.e., Trials $8,10,12,14,16$, and 18 ) to verify acquisition. Since the subjects could sample from both zones, then mob the stocked zone after reward discovery, we investigated response allocations for the entire duration, as well as the first 2 min, of trials. The latter, more conservative time frame was most likely to capture initial discrimination. We conducted analyses for the whole group, subgroups, and individuals, reporting varying degrees of freedom accordingly.

Analyses used UNIX/STAT compact data analysis programs (Perlman, 1986), with significance set at alpha $\leq .05$. Differences in group proximity, and in mean duration and frequency allocations, were assessed using two-tailed $t$ tests for related measures. We addressed within-subjects behavioral allocations across early and late trials using two-factor analyses of variance (ANOVAs) and $f$ tests for simple effects. We assessed individual frequency allocations using two-tailed goodness-of-fit tests ( $G$ test; Sokal \& Rohlf, 1981). Behavior allocations were also converted into difference scores (i.e., stocked value - nonstocked value) that reflect the magnitude of any preference. A positive value reflects a stocked-zone preference, a negative value reflects a nonstockedzone preference, and a zero value reflects no preference. The influence of reversal on difference scores, and on group proximity, was evaluated by one-way ANOVAs and Newman-Keul's ranked pairwise comparisons (Bruning \& Kintz, 1977). We compared changes in reward retrievals across trials within series using the test for significance of difference between two proportions (Bruning \& Kintz, 1977). For this test, a value of $z \geq 1.96$ or $\leq-1.96$ is significant at the .05 level, using a two-tailed test.

\section{RESULTS}

\section{Assessment of Possible One-Trial Learning Versus Gradual Learning of the Initial Discrimination}

The animals retrieved $88 \%$ of the available rewards on Trial 1 of the initial discrimination series. Despite this high success rate, group proximity during Trial 2 showed no zone preference: Equal numbers of animals entered both zones in the first $2 \min$ of Trial $2[t(7)=0$, n.s.], even though they had discovered $24 \%$ of the rewards. Furthermore, group proximity still showed no difference after $10 \mathrm{~min}$ into Trial $2[t(39)=0.49$, n.s.], despite the discovery of $65 \%$ of the rewards. Specifically, for those subjects rewarded in Trial $1(n=17), 11(65 \%)$ first entered the stocked zone and $6(35 \%)$ first entered the nonstocked zone on Trial 2, showing no significant difference $[G(1)=1.49$, n.s. $)$.

Thus, group proximity provided no evidence of onetrial learning during the initial acquisition series. Instead, this measure revealed gradual improvement as, over the entire series, more subjects entered the correct zone and fewer entered the incorrect zone (Figure 2). In early trials, equal numbers entered the two zones, both throughout trials $[t(4)=1.13$, n.s. $]$ and in the first $2 \mathrm{~min}$ of trials $[t(4)=0.66$, n.s. $]$ (Figure $2 a$ ). By late trials, however, more subjects entered the stocked zone, not only throughout trials $[t(4)=4.92, p<.01]$ but also in the first $2 \min [t(4)=5.30, p<.01]$ (Figure $2 b$ ).

Behavior allocations similarly showed no evidence of early preferences. On average, the subjects allocated their responses equally between zones, not only during Trial $2[t \mathrm{~s}(73)<1.17$, n.s.] but for at least the first 5 (early) acquisition trials $[t \mathrm{~s}(73)<1.92$, n.s.]. By late trials, however, mean responses reflected a preference for the stocked side on three measures [time in zones, zone entries, and box visits; $t \mathrm{~s}(73)>2.02, p \mathrm{~s}$ $<.05]$.

Because mean performance might mask individual preferences, we investigated individual response frequencies during the first 2 min of trials. In early trials, most animals ( $>88 \%$ ) showed no.preferences for stocked versus nonstocked sites, and, of the few that showed preferences, a comparable number preferred each zone $[G s(1)<2.77$, n.s.] (Figure 3a). By contrast, in late trials, not only did more animals show preferences but the overwhelming majority preferred stocked sites $[G \mathrm{~s}(1)>$ (a)

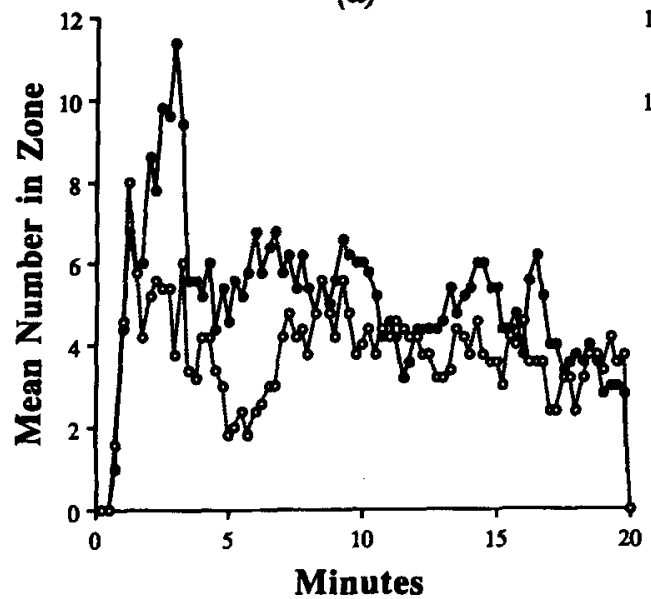

(b)

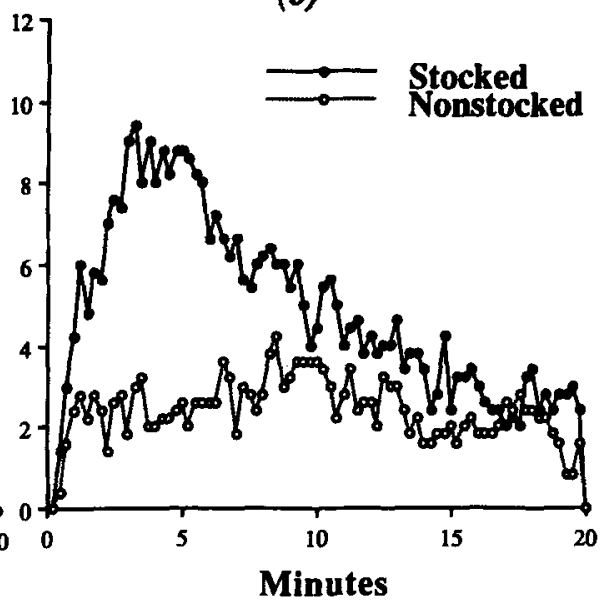

Figure 2, Mean number of subjects present in the stocked and nonstocked zones in 15-sec intervals during (a) early trials and (b) late trials of the initial discrimination series. 


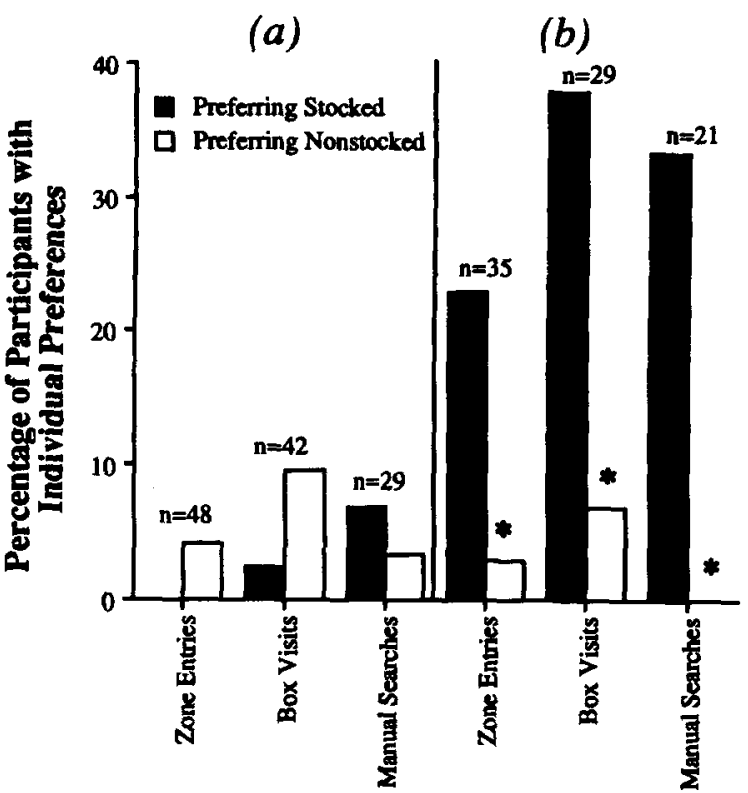

Figure 3. Percentage of participants showing significant individual preferences, on the three frequency measures, during the first 2 min of (a) early and (b) late trials of the initial discrimination series. The number of participants is given for each measure. ${ }^{*} p<.02$.

$6.20, p s<.02$ ] (Figure 3b). Thus, individual data, like group performance, showed gradual improvement.

Since, in the previous analysis, numbers and identities of participants varied across trials, we analyzed the behavior of the subset of subjects that participated in both early and late trials. Results were the same for all duration and frequency measures, so we report only the duration measures (Table 1). This subset showed constant participation across trials as, overall, subjects spent equal time in zones and on boxes; however, the amount of time allocated to the stocked versus nonstocked side varied between early and late trials [in-zone interaction, $F(1,30)=7.79, p<.01$; on-box interaction, $F(1,27)=$ $5.16, p<.05]$. As shown in Table 1, the subjects preferred the stocked side only in late trials. Though stocked values increased from early to late trials, these trends were not statistically reliable. There was, however, a significant decrease, from early to late trials, in time spent in the nonstocked zone.

Success in reward retrieval also indicated gradual improvement: The subjects retrieved an average of $85 \%$ of available rewards in early trials and $94 \%$ in late trials $(z=4, p<.05)$. Improved efficiency in reward retrieval was evident within the first $2 \mathrm{~min}$, increasing from $16 \%$ in early trials to $28 \%$ in late trials $(z=4, p<.05)$.

Since a substantial percentage of rewards was retrieved in the first 2 min of late trials, improvement in response allocations across trials was not a pure measure of discrimination: The animals could have entered the stocked zone simply because others were retrieving rewards and not because they were using the color cue. We addressed this problem by comparing zone selection in late trials before any reward was discovered. This encompassed only the first $30 \mathrm{sec}$ of trials and necessarily reduced the number of subjects available for analysis. Nevertheless, those animals entered the stocked zone more often than the nonstocked zone $[t(3)=5.20, p<$ $.025]$, suggesting that at least some subjects were attending to visual properties of the boxes.

\section{Assessment of Possible Rapid Learning Versus Gradual Learning-Set Formation Across Reversals}

Group proximity showed no evidence of rapid learning during the reversal series. For instance, of the animals that were rewarded on Trial 1 of the first reversal $(n=5)$, all $(100 \%)$ entered the wrong zone first on Trial $2[G(1)=6.93, p<.05]$. Similarly, of the 9 subjects rewarded on Trial 1 of the second reversal, $4(44 \%)$ entered the stocked zone first and $5(56 \%)$ entered the nonstocked zone first on Trial $2[G(1)=0.11$, n.s.]. Finally, of the 7 subjects rewarded on Trial 1 of the third reversal, $1(14 \%)$ entered the stocked zone first and $6(86 \%)$ entered the nonstocked zone first on Trial $2[G(1)=$ $3.96, p<.05]$. As expected from negative transfer, errors in group proximity increased immediately after a change in contingencies and, in the first reversal, persisted for at least four trials $[t(3)=4.84, p<.025]$. Although the effects of negative transfer were evident in the next two reversals (data not shown), errors of group proximity decreased across reversals as fewer subjects entered the wrong zone immediately after contingencies changed $[F(2,6)=18.80, p<.005]$.

Behavior allocations during reversals also showed no evidence of rapid learning. A global comparison of performance in each reversal used the average responses of the whole group $(N=74)$ across the entire duration of all trials analyzed. The number of measures on which the subjects showed significant preferences for the stocked side increased from two in the first reversal [time in zones and zone entries, $t \mathrm{~s}(73)>2.29, p \mathrm{~s}<.025$ ] to three

Table 1

Participant Duration Allocations $(M \pm S E M$ in Seconds) Between Zones During the First 2 Min of Early and Late Trials of the Initial Discrimination Series

\begin{tabular}{|c|c|c|c|c|c|}
\hline & \multicolumn{2}{|c|}{ Early (E) } & \multicolumn{2}{|c|}{ Late (L) } & \multirow[b]{2}{*}{$F(\mathrm{E}$ vs. $\mathrm{L})$} \\
\hline & $M$ & $S E M$ & $M$ & $S E M$ & \\
\hline \multicolumn{6}{|c|}{ Time in Zones } \\
\hline Overall $(\mathrm{S}+\mathrm{N})$ & 10.40 & 0.82 & 10.10 & 1.43 & 0.03 \\
\hline Stocked (S) & 10.56 & 1.15 & 13.63 & 2.07 & $3.24^{*}$ \\
\hline Nonstocked (N) & 10.24 & 1.19 & 6.56 & 1.78 & $4.61 \dagger$ \\
\hline$F(\mathrm{~S}$ vs. N $)$ & & & & & \\
\hline \multicolumn{6}{|c|}{ Time on Boxes } \\
\hline Overall $(\mathrm{S}+\mathrm{N})$ & 5.29 & 0.64 & 5.84 & 1.07 & 0.29 \\
\hline Stocked (S) & 4.97 & 0.82 & 7.74 & 1.57 & $4.01^{*}$ \\
\hline Nonstocked $(\mathrm{N})$ & 5.61 & 1.00 & 3.94 & 1.38 & $1.46 \dagger$ \\
\hline$F(\mathrm{~S}$ vs. $\mathrm{N})$ & \multicolumn{2}{|c|}{0.22} & \multicolumn{2}{|c|}{$7.53+$} & \\
\hline
\end{tabular}

Note-For "Time in Zones," $n=31$, and $d f=1,30$. For "Time on Boxes," $n=28$, and $d f=1,27 .{ }^{*} p<.10 . \quad \nmid p<.05 . \quad \ddagger p<.025$, $\S p<.001$. 
in the second reversal [time in zones, zone entries, and box visits, $t \mathrm{~s}(73)>2.15, p \mathrm{~s}<.05]$ to all five by the third reversal $[t \mathrm{~s}(73)>2.22, p \mathrm{~s}<.05]$.

To assure that global improvement did not reflect a decrease in participation by animals that were not learning, further analyses used the subset of animals $(n=57)$ that participated (i.e., entered zones more than once) in all series. Analyses of difference scores across reversals revealed increased stocked-zone preferences for all frequency measures $[F \mathrm{~s}(2,112)>4.46, p \mathrm{~s}<.02]$, with stronger preferences for provisioned sites in the second and third reversal than in the first $(p \mathrm{~s}<.05)$. Although duration measures showed the same pattern, the increases in stocked-zone preferences were not statistically reliable $\left[F_{\mathrm{s}}(2,112)<2.88, p \mathrm{~s} \geq .06\right.$, n.s.]. Thus, progressive improvement across reversals was reflected in the magnitude of correct responding by those participating in all series. Difference scores for box visits by this subset, during the first $2 \mathrm{~min}$ of the second and last trials only, are presented for all series, including the initial discrimination, for purpose of comparison (Figure 4). Acquisition of each problem was evidenced by improved performance within series (depicted by lasttrial positive preferences) and by negative transfer across series following contingency reversals (depicted by Trial 2 errors).

Finally, within each reversal, the subjects showed gradual improvement in reward retrieval efficiency, since rate of success increased from early to late trials $(z \mathrm{~s}>4, p \mathrm{~s}<.05)$. To compare across reversals, we defined retrieval efficiency as the percentage, from the daily total of rewards obtained, that were discovered each minute (Figure 5). In the first and second reversal, improvement in retrieval efficiency during the first 5 min of trials was gradual across trials (Figures -5a and 5 b); however, by the third reversal, efficiency approached the maximum after only one trial (Figure $5 \mathrm{c}$ ).

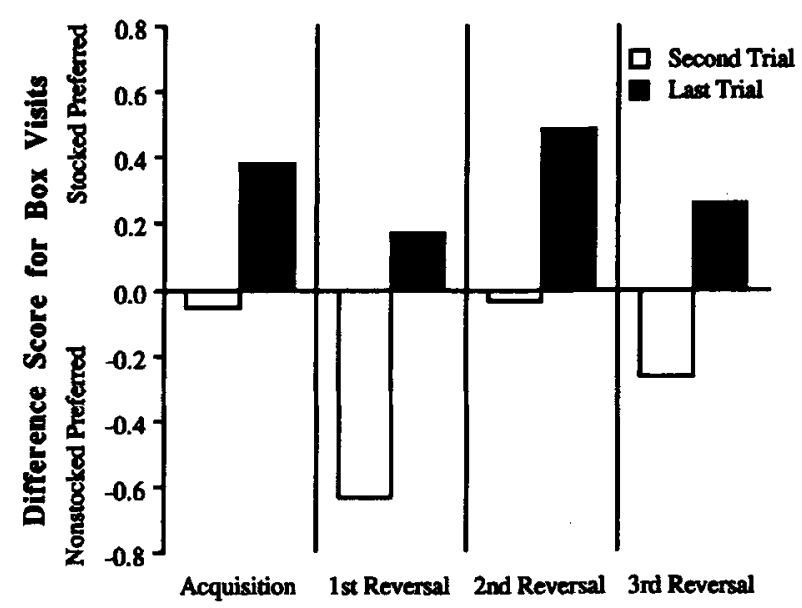

Figure 4. Second- and last-trial performance in each series, expressed as the difference between stocked and nonstocked box visits. The behavior of subjects $(n=57)$ participating in all series is shown for the first $2 \mathrm{~min}$ of selected trials.

\section{DISCUSSION}

When a foraging troop of monkeys encounters a feeding area containing various food signs (Jarvik, 1953; Menzel, 1974), animals discriminate between choice sites and less desirable sites, with successful individuals depleting the preferred resources. In the present study, rhesus monkeys were exposed to a series of discrimination problems that simulated this foraging situation. The use of an ecologically relevant task, presented in a socially relevant context, required important differences in procedure relative to traditional studies. For instance, during each trial, the animals were tested as a group, rather than individually. Also, the animals could respond more than once and were permitted to correct their choices within trials. Moreover, the probability of being rewarded was variable and decreased with time in a given trial. Finally, several different dependent measures were used to evaluate performance. Despite these procedural differences, mean and individual performance during an initial acquisition phase and across a later reversal phase improved gradually, suggesting incremental learning. Thus, results show that, in solving certain complex tasks, animals require a period of "learning how to learn" (Harlow, 1949).

Although progressive improvement within problems does not necessarily reflect the acquisition of a new ability, incremental learning during the initial acquisition phase is inconsistent with the notion that progressive improvement reflects the regaining of previous levels of efficiency after situation-induced negative transfer. The effects of negative transfer were present, however, during the reversal phase. The increase in errors following each reversal of contingencies reflected tendencies to explore the colored boxes formerly associated with rewards and provided the strongest evidence for previous discrimination acquisition.

The pattern of gradual improvement reported here is consistent with the pattern of incremental learning described in traditional studies of individual discrimination learning (Fobes \& King, 1982; Harlow, 1944, 1949); yet, it differs from the pattern of rapid learning reported in group studies (Lepoivre \& Pallaud, 1986; Menzel \& Juno, 1982; Pallaud \& Lepoivre, 1985). Thus, discrimination testing of primates under more natural conditions produces both rapid and gradual learning, depending on, as yet, unidentified factors. Results of the present study cannot be easily attributed to the arbitrary nature of the task, since it shared important features with challenges faced by foraging animals. Unlike Lepoivre and Pallaud (1986), we did not find that choice of dependent variable influenced the pattern of learning observed. In the present study, all variables, including the number of subjects responding, their individual behavior, and their success rate, consistently showed gradual improvement.

Direct comparison across the three studies of discrimination learning in a group context is not possible; yet, several important procedural differences may pro- 

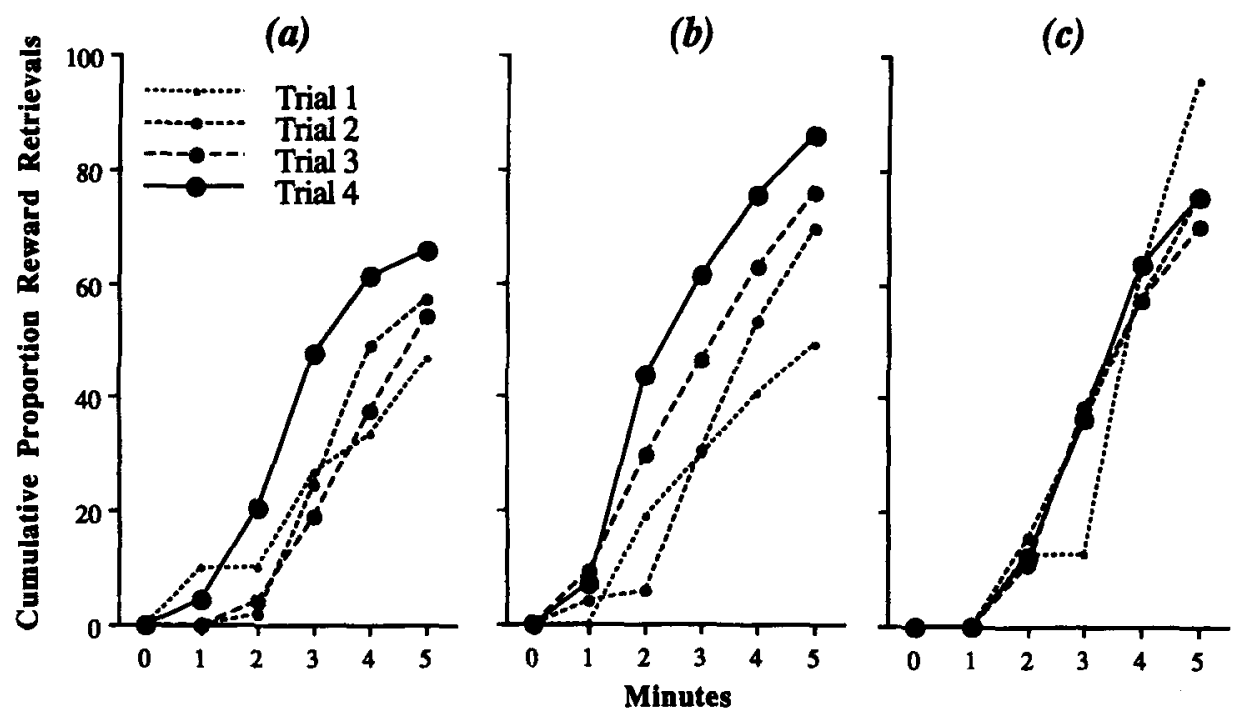

Figure 5. Cumulative proportions of rewards retrieved across the first 5 min of Trials $1-4$ for the (a) first, (b) second, and (c) third reversals.

vide explanations for the observed performance patterns. As previously discussed (Schrier \& Thompson, 1984), results may relate to the paradigms used, since successive versus simultaneous presentation can yield contrasting results (Fobes \& King, 1982; Mackintosh, 1974). Similarly, differences may relate to the task or method of presentation, with the speed of learning reflecting differences in perceived complexity. For instance, animals require only one or two trials to learn the relationship between a stimulus and a reward when the cue is closely associated with the reward, but they require several trials if the cue is spatially removed from the reward (Jarvik, 1953). In Menzel and Juno's (1982) task, the cue and the reward were tightly coupled, whereas in the present task, they were not.

Alternately, rapid learning by an 8-member family group of marmosets (Menzel \& Juno, 1982) and an 11member baboon group (Lepoivre \& Pallaud, 1986), compared with gradual learning by our troop of 74 rhesus macaques, may reflect species-specific characteristics of learning or aspects of social organization. Although differences may simply show that group measures poorly reflect individual performance unless the numbers contributing to those measures are sufficiently large, they may also show that information about the appropriate stimuli disperses less rapidly in larger groups. If the latter were true, group measures might underestimate the performance of certain individuals. Since, in the present study, individual performance was consistent with mean performance, gradual improvement is not solely explained by attentional issues or competition associated with large groups.

Instead, group size or composition may influence which learning strategies are used. The complex environment of group tests provides several potentially relevant cues, including the behavior of others, in addition to the discriminative stimulus. Because, in our task, rewards became depleted, a premium was placed on the ability to rapidly select the most salient cue (in this case, box color). The animals first entering the stocked zone during late trials showed strong evidence of color discrimination acquisition. Latecomers, on the other hand, may have used a less profitable strategy, basing their choice on the behavior of others who discovered rewards. As groups get larger, the number of potentially relevant cues increases, diluting the potency of any one cue, resulting in animals within a group using different cues to solve the task at hand.

In this regard, it is striking that the only measure suggesting one-trial learning in social groups is proximity, a measure for which the behavior of others can be easily observed. In contrast, measures of individual success, or potential success, reflect gradial learning whether or not the subject is tested in a group or singly. Thus, it may be that discrimination based on attending to social behavior is rapidly acquired in primates, whereas learning based on inanimate cues is gradually acquired. The resolution of this issue requires more extensive focus on learning in social groups. Whereas our results suggest that ecological relevance and social influence alone cannot account for the rapid learning reported in previous group tests, the specific factors responsible remain elusive. It is, however, apparent that gradual learning is one process used in social groups of rhesus monkeys to acquire meaningful discriminations.

\section{REFERENCES}

Bruning, J. L., \& Kintz, B. L. (1977). Computational handbook of statistics (2nd ed.). Glenview, IL: Scott, Foresman.

D' Amato, M. R., \& Fazzaro, J, (1966). Attention and cue-producing behavior in the monkey. Journal of the Experimental Analysis of Behavior, 9, 469-473. 
De Valois, R. L., \& JaCobs, G. H. (1968). Primate color vision. Science, 162, 533-540.

Devine, J. V. (1970). Stimulus attributes and training procedures in learning-set formation of rhesus and cebus monkeys. Journal of Comparative \& Physiological Psychology, 73, 62-67.

FoBes, J. L., \& KING, J. E. (1982). Measuring primate learning abilities. In J. L. Fobes \& J. E. King (Eds.), Primate behavior (pp. 289326). New York: Academic Press.

GouLD, J. L., \& MARLER, P. (1984). Ethology and the natural history of learning. In P. Marler \& H. S. Terrace (Eds.), The biology of learning (pp. 47-74). New York: Springer-Verlag.

HaRLow, H. F. (1944). Studies in discrimination learning by monkeys: I. The learning of discrimination series and the reversal of discrimination series. Journal of General Psychology, 30, 3-12.

HaRLow, H. F. (1949). The formation of learning sets. Psychological Review, 56, 51-65.

JARVIK, M. E. (1953). Discrimination of colored food and food signs by primates. Journal of Comparative \& Physiological Psychology, 46, 390-392.

JAY, P. (1965). Field studies. In A. M. Schrier, H. F. Harlow, \& F. Stollnitz (Eds.), Behavior of nonhuman primates (Vol. 2, pp. 525-591). New York: Academic Press.

Johnston, T. D. (1981). Contrasting approaches to a theory of learning. Behavioral \& Brain Sciences, 4, 125-139.

JohNSTON, T. D. (1982). Selective costs and benefits in the evolution of learning. In J. S. Rosenblatt, R. A. Hinde, C. Beer, \& M. C. Busnel (Eds.), Advances in the study of behavior (Vol. 12, pp. 65-106). New York: Academic Press.

Jolly, A. (1985). The evolution of primate behavior. American Scientist, 73, 230-239.

KAMIL, A. C., \& YoerG, S. I. (1982). Learning and foraging behavior. In P. P. G. Bateson \& P. H. Klopfer (Eds.), Perspectives in ethology (Vol. 5, pp. 325-364). New York: Plenum Press.

Lepoivre, H., \& Pallaud, B. (1985). Social facilitation in a troop of Guinea baboons (Papio papio) living in an enclosure. Behavioural Processes, 11, 405-418.

Lepoivre, H., \& Pallaud, B. (1986). Learning set formation in a group of Guinea baboons (Papia papio): Importance of the behavioral variable measured. American Journal of Primatology, 10, 25-36.

LEVINE, M. (1959). A model of hypothesis behavior in discrimination learning set. Psychological Review, 66, 353-366.
LINDBURG, D. G. (1971). The rhesus monkey in North India: An ecological and behavioral study. Primate Behavior, 2, 1-106.

Mackintosh, N. J. (1974). The psychology of animal learning (pp. 543-619). New York: Academic Press.

Menzel, E. W., JR. (1974). A group of young chimpanzees in a one acre field. In A. M. Schrier \& F. Stollnitz (Eds.), Behavior of nonhuman primates: Modern research trends (Vol. 5, pp. 83-149). New York: Academic Press.

MENZEL, E. W., JR., \& JUno, C. (1982). Marmosets (Saguinus fuscicollis): Are learning sets learned? Science, 217, 750-752.

Menzel, E. W., JR., \& Juno, C. (1984). Are learning sets learned? Or: Perhaps no nature-nurture issue has any simple answer. Animal Learning \& Behavior, 12, 113-115.

Pallaud, B., \& Lepoivre, H. (1985). Apprentissage d'inversion dans une situation de discrimination spatiale par un groupe de babouins: Interactions entre facteurs cognitifs et facteurs sociaux. Cahiers de Psychologie Cognitive, 5, 527-551.

PERLMAN, G. (1986). UNIX/STAT manuals: Data analysis programs on UNIX and MSDOS. Tyngsboro, MA: Wang Institute.

SCHRIER, A. M. (1984). Learning how to learn: The significance and current status of learning set formation. Primates, 25, 95-102.

SCHRIER, A. M., \& ThOMPson, C. R. (1984). Are learning sets learned? A reply. Animal Learning \& Behavior, 12, 109-112.

Seligman, M. E. P. (1970). On the generality of the laws of learning. Psychological Review, 77, 406-418.

SHETTLEWORTH, S. J. (1984). Learning and behavioural ecology. In J. R. Krebs \& N. B. Davies (Eds.), Behavioural ecology: An evolutionary approach (pp. 170-194). Sunderland, MA: Sinauer.

SNODDERLY, D. M. (1979). Visual discriminations encountered in food foraging by a neotropical primate: Implications for the evolution of color vision. In E. H. Burtt, Jr. (Ed.), The behavioral significance of color (pp. 237-279). New York: Garland STPM Press.

SOKAL, R. R., \& RoHLF, J. F. (1981). Biometry: The principles and practice of statistics in biological research (2nd ed.). San Francisco: W. H. Freeman.

TimberLaKE, W. (1990). Natural learning in laboratory paradigms. In D. A. Dewsbury (Ed.), Contemporary issues in comparative psychology (pp. 31 -54). Sunderland, MA: Sinauer.

(Manuscript received January 13, 1993; revision accepted for publication May 14, 1994.) 